\title{
INFINITE MATRICES AND ABSOLUTE ALMOST CONVERGENCE
}

\author{
MURSALEEN \\ Department of Mathematics \\ Aligarh Muslim University \\ Aligarh - 202001, INDIA \\ (Received January 26, 1981 and in revised form August 16, 1982)
}

ABSTRACT. In 1973, Stieglitz [5] introduced a notion of $F_{B}$-convergence which provided a wide generalization of the classical idea of almost convergence due to Lorentz [1]. The concept of strong almost convergence was introduced by Maddox [3] who later on generalized this concept analogous to Stieglitz's extension of almost convergence [4]. In the present paper we define absolute $F_{B}$-convergence which naturally emerges from the concept of $\mathrm{F}_{\mathrm{B}}$-convergence.

KEY WORDS AND PHRASES. Infinite matrices, almost convergence, strong almost convergence, $F_{B}$-convergence, absolute $F_{B}$-convergence.

1980 MATHEMATICS SUBJECT CLASSIFICATION CODES. $40 \mathrm{CO5}, 40005$.

\section{INTRODUCTION.}

Let $l_{\infty}, c$, and $c_{0}$ denote respectively the Banach spaces of bounded, convergent, and null sequences $x=\left(x_{k}\right)$ of complex numbers with norm ||$x||=\sup _{k}\left|x_{k}\right|$, and 1et $v$ be the space of sequences of bounded variation, that is,

$$
\mathrm{v}=\left\{\mathrm{x}: \quad|| \mathrm{x}|| \equiv \sum_{\mathrm{k}=0}^{\infty}\left|\mathrm{x}_{\mathrm{k}}-\mathrm{x}_{\mathrm{k}-1}\right|<+\infty, \mathrm{x}_{-1}=0\right\} \text {. }
$$

Suppose that $B=\left(B_{i}\right)$ is a sequence of infinite complex matrices with $B_{i}=\left(b_{n p}(i)\right)$. Then $x \in \ell_{\infty}$ is said to be $F_{B}$-convergent [5], to the value Lim $B x$, if

$$
\lim _{n \rightarrow \infty}\left(B_{i} x\right)_{n}=\lim _{n \rightarrow \infty} \sum_{p=0}^{\infty} b_{n p}(i) x_{p}=\operatorname{Lim} B x,
$$

uniformly for $i=0,1,2, \ldots$.

The space $\mathrm{F}_{\mathrm{B}}$ of $\mathrm{F}_{\mathrm{B}}$-convergent sequences depends on the fixed chosen sequence $B=\left(B_{i}\right)$. In case $B=B_{0}=$ (I) (unit matrix), the space $F_{B}$ is same as $c$ and, for 
$B=B_{1}=\left(B_{i}^{(1)}\right)$, it is same as the space $f$ of almost convergent sequences [1], where $\mathrm{B}_{i}^{(1)}=\left(\mathrm{b}_{\mathrm{np}}^{(1)}(\mathrm{i})\right)$ with

$$
b_{n p}^{(1)}(i)=\left\{\begin{array}{ll}
\frac{1}{n+1}, & i \leq p \leq i+n \\
0 & \text { elsewhere }
\end{array}\right\}
$$

Maddox [4] generalized strong almost convergence by saying that $x_{p} \rightarrow s\left[F_{B}\right]$ if and only if

$$
\sum_{p} b_{n p}(i)\left|x_{p}-s\right| \rightarrow 0(n \rightarrow \infty \text {, uniformly in } i)
$$

assuming that the series in (1.1) converges for each $n$ and $i$.

In particular, if $B=B_{0}$, the $\left[F_{B}\right]=c$; if $B=B_{i}$, then $\left[F_{B}\right]=[f]$, the space of strongly almost convergent sequences [3]. We shall write $e_{k}=(0,0, \ldots, 0,1$ (kth entry), $0, \ldots)$ and $e=(1,1,1, \ldots)$.

Let $\mathbf{s}$ be the space of all complex sequences and

$$
\begin{aligned}
d_{B}=\{x \in s: \quad \operatorname{Lim} B x & \left.=\underset{n \rightarrow \infty}{\lim }\left(B_{i} x\right)_{n} \text { exists for each } i\right\} \\
F_{B}=\left\{x \in\left(d_{B} \cap \ell_{\infty}\right):\right. & \begin{array}{r}
\lim _{n \rightarrow \infty} t_{n}(i, x) \text { exists uniform1y in } i, \\
\text { and the 1imit is independent of } i\},
\end{array}
\end{aligned}
$$

where

$$
t_{n}(i, x)=\left[\begin{array}{ll}
\sum_{p=j}^{\infty} b_{n p}(i) x_{p}, & (n \geq 1) \\
\sum_{i=0}^{\infty} \beta_{0 p}(i) x_{p}, & (n=0) \\
0 \quad & (n=-1)
\end{array}\right]
$$

and

$$
\beta_{0 p}(i)=\left\{\begin{array}{ll}
1 & \text { if } p=i \\
0 & \text { otherwise. }
\end{array}\right\}
$$

Let

$$
\emptyset_{n}(i, x)=t_{n}(i, x)-t_{n-1}(i, x)
$$

Therefore, we have

$$
\emptyset_{n}(i, x)=\left[\begin{array}{ll}
\sum_{p=0}^{m}\left[b_{n p}(i)-b_{n-1, p}(i)\right] x_{p}, & (n \geq 1) \\
\sum_{p=0}^{\infty} \beta_{0 p} \text { (i) } x_{p}, \quad(n=0)
\end{array}\right]
$$


DEFINITION. Let $B=\left(B_{i}\right)$ be a sequence of infinite matrices with $B_{i}=\left(b_{n p}(i)\right)$. A sequence $x \in \ell_{\infty}$ is said to be absolutely $F_{B}$-convergent if $\sum_{n=0}^{\infty}\left|\emptyset_{n}(i, x)\right|$ converges uniformly for $i \geq 0$, and $\lim _{n \rightarrow \infty} t_{n}(i, x)$ which must exist should take the same value for al1 i. We denote the space of absolute $F_{B}$-convergent sequences by $v(B)$.

2. THE MAIN RESULT.

In this note, we denote by $(v, v(B))$ the set of matrices which give new classes of absolute B-conservative matrices and absolute almost B-conservative matrices.

Let $\mathrm{A}$ be any infinite complex matrix for which the pth row-sum converges for a given $\mathrm{x}$ for all $\mathrm{x}$ in some class.

We have

$$
A_{p} x=(A x)_{p}=\sum_{k=0}^{\infty} a_{p k} x_{k}
$$

and

Therefore,

$$
\left(B_{i}\right)_{n}=\sum_{p=0}^{j} b_{n p}(i) x_{p} \text {. }
$$

$$
\begin{aligned}
\left(B_{i} A x\right)_{n} & =\sum_{p=0}^{\infty} b_{n p} \text { (i) } A_{p} x \\
& =\sum_{p=0}^{\infty} b_{n p} \text { (i) } \sum_{k=0}^{\infty} a_{p k} x_{k},
\end{aligned}
$$

and, assuming the interchange of order of summation can be justified (see lemma), we get that

$$
\left(B_{i} A x\right)_{n}=\sum_{k=0}^{\infty} \sum_{p=0}^{n} b_{n p} \text { (i) } a_{p k} x_{k}
$$

Now, by (1.2) and (2.1), we have

$$
\begin{aligned}
\emptyset_{n}(i, A x) & =t_{n}(i, A x)-t_{n-1}(i, A x) \\
& = \begin{cases}\sum_{p=0}^{\infty}\left[b_{n p}(i)-b_{n-1, p}(i)\right] A_{p} x, \quad(n \geqslant 1), \\
\sum_{p=0}^{n} \beta_{0 p} \text { (i) } A_{p} x & (n=0), \\
& =\sum_{k=0}^{\infty} g_{n k} \text { (i) } x_{k},\end{cases}
\end{aligned}
$$

where 


$$
g_{n k}(i)=\begin{array}{ll}
\sum_{p=0}^{\infty}\left[b_{n p}(i)-b_{n-1, p}(i)\right] a_{p k}, & (n \geq 1), \\
\sum_{p=0}^{\infty} \beta 0 p & (i) a_{p k}, \quad(n=0) .
\end{array}
$$

THEOREM. Let $B=\left(B_{i}\right)$ be a sequence of infinite matrices with

$$
\sup _{n} \sum_{p=0}^{\infty}\left|b_{n p}(i)\right|<\infty, \quad \text { for each } i \text {. }
$$

Let $A$ be an infinite matrix. Then $A: v \rightarrow v(B)$ if and only if

(i) $\sup _{\mathrm{p}, \mathrm{k}}\left|\sum_{\ell=\mathrm{k}}^{n} \mathrm{a}_{\mathrm{p} \ell}\right|<\infty$,

(ii) there is an $\mathrm{N}$ such that for $\mathrm{r}, \mathrm{i}=0,1,2, \ldots$

$$
\sum_{n=N}^{\infty}\left|\sum_{k=0}^{r} g_{n k}(i)\right| \leq k \quad \text { (constant), }
$$

(iii) $\left(a_{p k}\right)_{p \geq 0} \in v(B)$ for each $k$, and

$$
\text { (iv) }\left(\sum_{\mathrm{k}=0}^{\infty} a_{\mathrm{pk}}\right)_{\mathrm{p} \geq 0} \in \mathrm{v}(\mathrm{B}) \text {. }
$$

Let $A \in(v, v(B))$. For each $k$, let $a_{p k}$ be $F_{B}$-convergent with limit $\alpha_{k}$. And let $\sum_{k=0}^{\infty} a_{p k}$ be $F_{B}$-convergent with 1imit $\alpha$. (In each case, limit is taken for $p \geq 0$ ). If $x=\left(x_{k}\right) \in v$, then

$$
\lim _{n \rightarrow \infty} t_{n}(i, A x)=\alpha \lim _{k \rightarrow \infty} x_{k}+\sum_{k=0}^{\infty}\left(x_{k}-\lim _{k \rightarrow \infty} x_{k}\right) \alpha_{k} .
$$

We use the following lemma in the proof.

LEMMA. If either the necessity part or the sufficiency part of the theorem holds, then, for $\mathrm{x} \in \mathrm{v}$,

$$
\sum_{p=0}^{\infty} b_{n p}(i) \sum_{k=0}^{\infty} a_{p k} x_{k}=\sum_{k=0}^{\infty} x_{k} \sum_{p=0}^{\infty} b_{n p}(i) a_{p k} \cdot
$$

PROOF. If either $A: \quad v \rightarrow v(B)$ or the conditions (i)-(iv) of the theorem hold, then by parti 11 summation, for $\mathrm{x} \in \mathrm{v}$,

$$
\sum_{k=0}^{\infty} a_{p k} x_{k}=\sum_{k=0}^{\infty} d_{p k}\left(x_{k}-x_{k-1}\right)
$$

where $d_{p k}=\sum_{\ell=k}^{\infty} a_{p l}$. Since condition (i) holds, $d_{p k}$ is bounded for all $p, k$. Thus 


$$
\begin{aligned}
\sum_{p=0}^{\infty} b_{n p}(i) \sum_{k=0}^{\infty} a_{p k} x_{k} & =\sum_{p=0}^{\infty} b_{n p}(i) \sum_{k=0}^{\infty} d_{p k}\left(x_{k}-x_{k-1}\right) \\
& =\sum_{k=0}^{\infty}\left(x_{k}-x_{k-1}\right) \sum_{p=0}^{\infty} b_{n p}(i) d_{p k},
\end{aligned}
$$

(where the inversion is justified by absolute convergence)

$$
=\sum_{k=0}^{\infty} x_{k} \sum_{p=0}^{\infty} b_{n p}(i) a_{p k}
$$

since

$$
\lim _{k \rightarrow \infty} x_{k} \sum_{p=0}^{\infty} b_{n p}(i) d_{p k}=0 .
$$

PROOF OF THEOREM. Necessity. Condition (i) follows from the fact that $A: v \rightarrow l_{\infty}$. Since $e_{k}, e \in v$, necessity of (iii) and (iv) is obvious.

It is clear that, for fixed $p$ and $j$,

$$
\mathrm{x} \rightarrow \sum_{\mathrm{k}=0}^{\mathrm{j}} \mathrm{a}_{\mathrm{pk}} \mathrm{x}_{\mathrm{k}}
$$

is a continuous linear functional on $v$. We are given that, for all $x \in v$, it tends to a limit as $j \rightarrow \infty$ (for fixed $p$ ) and hence, by the Banach-Steinhaus Theorem [2], this limit $A_{p} x$ is also a continuous linear functional on $v$.

We observe that, although $\sum_{n=0}^{\infty}\left|\emptyset_{n}(i, A x)\right|$ is uniformly convergent in $i$, it needs not be uniformly bounded in $i$. For example, if $\emptyset_{0}(i, A x)=i$ and $\emptyset_{n}(i, A x)=0$ ( $n \geq 1$ and $i$ ), then $\sum_{n=0}^{\infty}\left|\emptyset_{n}(i, A x)\right|$ is uniformly convergent in $i \geq 0$ but not uniformly bounded. Now, we can say that uniforn convergence bears only on the behaviour of $\emptyset_{n}(i, A x)$ for sufficiently large $n$. Thus, by definition, there is an $m$ such that

$$
q_{m, i}(x)=\sum_{n=m}^{\infty}\left|\emptyset_{n}(i, A x)\right| .
$$

For $m \geq o, i \geq 0, q_{m, i}$ is a continuous seminorm on $v$, and there is an integer $\mathrm{N}$ such that $\left\{\mathrm{q}_{\mathrm{N}, \mathrm{i}}\right\}_{i \geq 0}$ is pointwise bounded on $v$. Such an $\mathrm{N}$ exists. For suppose not. Then for $r=0,1,2, \ldots$ ther exists $x_{r} \in v$ with

$$
\sup _{i \geq 0} q_{r, i}\left(x_{r}\right)=\infty .
$$

By the principle of condensation of singularities [6],

$$
\left\{x \in v: \sup _{i \geq 0} q_{r, i}(x)=\infty \quad \text { for } \quad r=0,1,2, \ldots\right\}
$$


is of second category in $\mathrm{v}$ and hence nonempty, i.e., there is $\mathrm{x} \in \mathrm{v}$ with

$$
\sup _{i \geq 0} q_{r, i}(x)=\infty \quad \text { for } \quad r=0,1,2, \ldots
$$

But this contradicts the fact that to each $x \in v$ there exists an integer $N_{x}$ with $\sup _{i \geq 0} q_{N}, i(x)<\infty$.

Now, by another application of the Banach-Steinhaus Theorem, there exists a constant $M$ such that

$$
\mathrm{q}_{\mathrm{N}, \mathrm{i}}(\mathrm{x}) \leq \mathrm{M}|| \mathrm{x}||
$$

Apply (2.3) with $x=\left(x_{k}\right)$ defined by $x_{k}=1$ for $k \leq r$ and o for $k>r$. Hence (ii) must hold.

Sufficiency. Suppose that the conditions (i)-(iv) hold and that $x \in v$. We have defined $v(B)$ as a subspace of $\ell_{\infty}$. Thus, in order to show that $A x \in v(B)$, it is necessary to prove that $\mathrm{Ax}$ is bounded. By virtue of condition (i), this follows immediately.

Now, it follows from (iv) and the lemma that

$$
\sum_{k=0}^{\infty} g_{n k}(i)
$$

converges for all $\mathrm{i}, \mathrm{n}$. Hence, if we write

$$
h_{n k}(i)=\sum_{\ell=k}^{\infty} g_{n \ell}(i),
$$

then $h_{n k}(i)$ is defined, also for fixed $i, n$,

$$
\mathrm{h}_{\mathrm{nk}}(\mathrm{i}) \rightarrow 0
$$

as $k \rightarrow \infty$. Now condition (iv) gives us that

$$
\sum_{n=0}^{\infty}\left|h_{n 0}(i)\right|
$$

converges iniformly in $i$, and, for suitable chosen $N$,

$$
\sum_{n=N}^{\infty}\left|h_{n 0}(i)\right|
$$

is bounded. By virtue of condition (iii), for fixed $k$, we get that

converges uniformly in i. Since

$$
\sum_{n=0}^{\infty}\left|g_{n k}(i)\right|
$$

$$
h_{n k}(i)=h_{n 0}(i)-\sum_{\ell=0}^{k-1} g_{n \ell}(i),
$$


it follows that, for fixed $k$,

$$
\sum_{n=0}^{\infty}\left|h_{n k}(i)\right|
$$

converges uniformly in $i$.

Now

$$
\begin{aligned}
\emptyset(\mathrm{i}, \mathrm{Ax}) & =\sum_{k=0}^{\infty} g_{n k}(i) x_{k} \\
& =\sum_{k=0}^{\infty}\left[h_{n k}(i)-h_{n, k+1} \text { (i)] } x_{k}\right. \\
& =\sum_{k=0}^{\infty} h_{n k}(i)\left(x_{k}-x_{k-1}\right),
\end{aligned}
$$

by $(2.4)$ and the boundedness of $x_{k}$.

Condition (ii) and the boundedness of (2.6) show that

$$
\sum_{n=N}^{\infty}\left|h_{n k}(i)\right|
$$

is bounded for all k,i. We can make

$$
\sum_{k=k_{0}+1}^{\infty}\left|x_{k}-x_{k-1}\right|
$$

arbitrarily small by choosing $k_{0}$ sufficiently large. It therefore follows that, given $\varepsilon>0$, we can choose $k_{0}$ so that, for all $i$,

$$
\sum_{n=N}^{\infty}\left|\sum_{k=k+1}^{\infty} h_{n k}(i)\left(x_{k}-x_{k-1}\right)\right|<\varepsilon .
$$

By the uniform convergence of $(2.8)$, it follows that, once $k_{0}$ has been chosen, we can choose $\mathrm{n}$ so that, for all $\mathrm{i}$,

$$
\sum_{n=n_{0}+1}^{\infty}\left|\sum_{k=0}^{k_{0}} h_{n k}(i)\left(x_{k}-x_{k-1}\right)\right|<\varepsilon .
$$

It follows from (2.11) that the same inequality holds when $\sum_{n=N}^{\infty}$ is replaced by $\sum_{n=n_{0}+1}^{\infty}$; hence, for all i,

$$
\sum_{n=n+1}^{\infty}\left|\sum_{k=0}^{\infty} h_{n k}(i)\left(x_{k}-x_{k-1}\right)\right|<2 \varepsilon .
$$

Hence,

$$
\sum_{n=n_{0}+1}^{\infty}\left|\emptyset_{n}(i, A x)\right|<2 \varepsilon .
$$

Thus

$$
\sum_{n=0}^{\infty}\left|\emptyset_{n}(i, A x)\right|
$$


converges uniformly.

Now, by virtue of $(2.9)$, we have

$$
\begin{aligned}
\lim _{n \rightarrow \infty} t_{n}(i, A x)-t_{N-1}(i, A x) & =\sum_{n=N}^{\infty} \sum_{k=0}^{\infty} h_{n k}(i)\left(x_{k}-x_{k-1}\right) \\
& =\sum_{k=0}^{\infty}\left(x_{k}-x_{k-1}\right) \sum_{n=N}^{\infty} h_{n k}(i)
\end{aligned}
$$

the assertion being justified by absolute convergence because of the boundedness of $(2.10)$. By $(2.7)$, we have

$$
\begin{aligned}
\sum_{n=N}^{\infty} h_{n k}(i) & =\sum_{n=N}^{\infty} h_{n o}(i)-\sum_{\ell=0}^{k-1} \sum_{n=N}^{\infty} g_{n \ell}(i) \\
& =\alpha-\sum_{\ell=0}^{k-1} \alpha_{\ell}-\sum_{\ell=k}^{\infty} \sum_{p=0}^{\infty} b_{N-1, p}(i) a_{p \ell} .
\end{aligned}
$$

Thus,

$$
\lim _{n \rightarrow \infty} t_{n}(i, A x)=\alpha \lim _{k \rightarrow \infty} x_{k}+\sum_{k=0}^{\infty}\left(x_{k}-\lim _{k \rightarrow \infty} x_{k}\right) \alpha_{k} .
$$

This completes the proof.

\section{REFERENCES}

1. LORENTZ, G.G. A contribution to the theory of divergent series, Acta Math. $\underline{80}$ (1948), 167-190.

2. MADDOX, I.J. Elements of Functional Analysis, Cambridge University Press, 1970.

3. MADDOX, I.J. A new type of convergence, Math. Proc. Camb. Phil. Soc. 83 (1978), 61-64.

4. MADDOX, I.J. On strong almost convergence, Math. Proc. Camb. Phil. Soc. 85 (1979), 345-350.

5. STIEGLITZ, M. Eine Verallgemeinerung des Begriffs der Fastkonvergenz, Math. Japon. 18 (1973), 53-70.

6. YOSIDA, K. Functional Analysis, Springer-Verlag, Berlin-Heidelberg-New York, 1966 


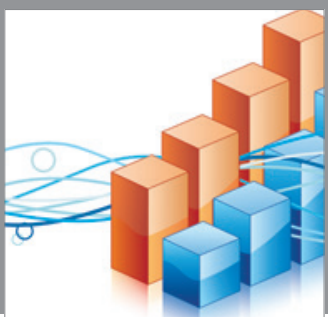

Advances in

Operations Research

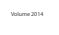

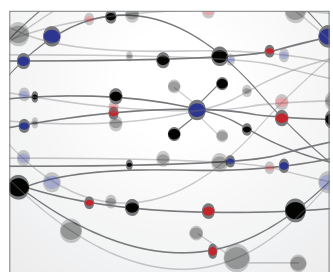

\section{The Scientific} World Journal
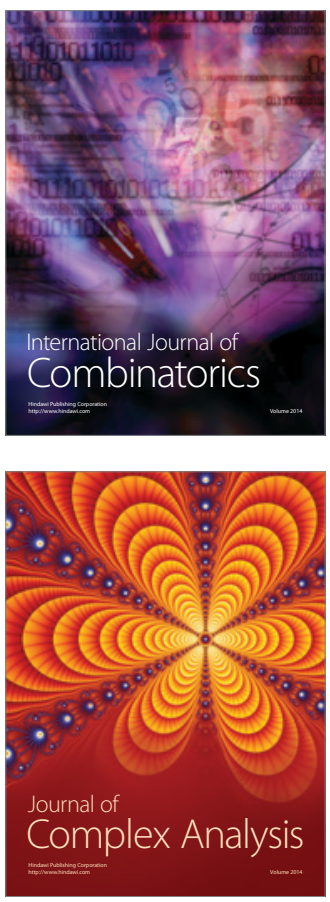

International Journal of

Mathematics and

Mathematical

Sciences
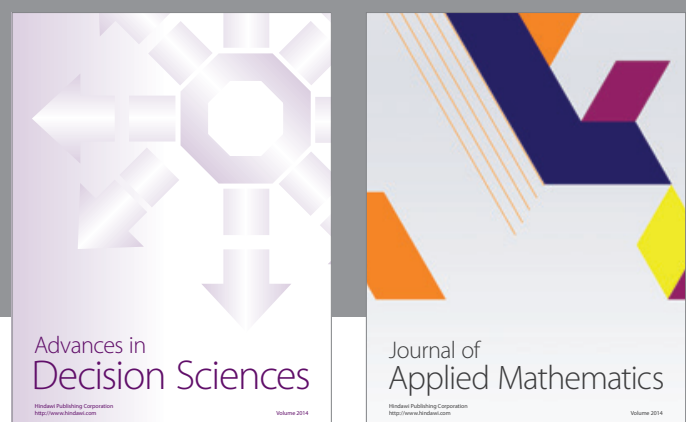

Journal of

Applied Mathematics
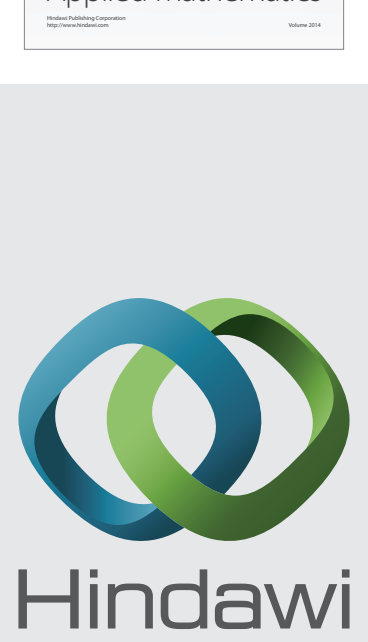

Submit your manuscripts at http://www.hindawi.com
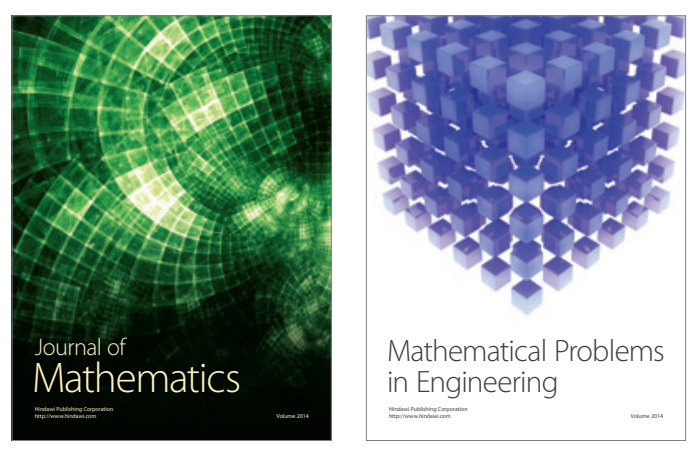

Mathematical Problems in Engineering
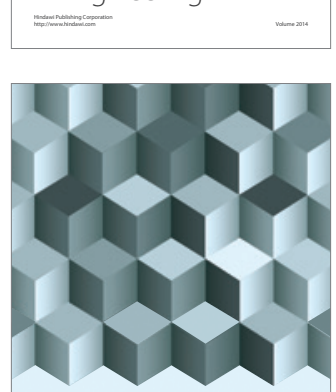

Journal of

Function Spaces
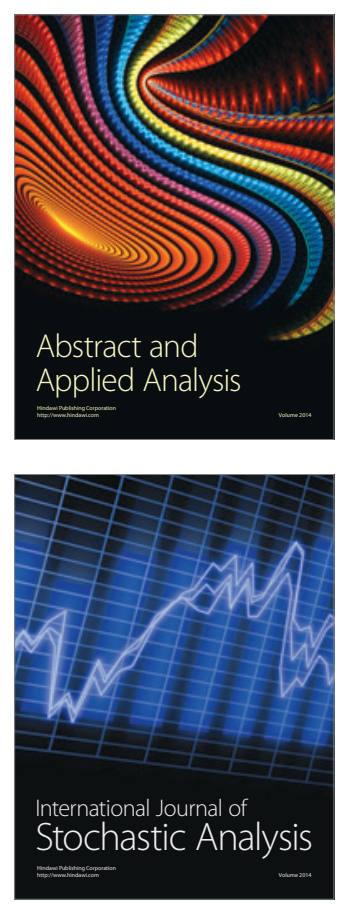

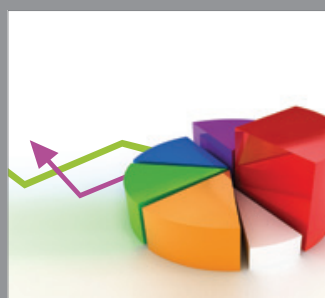

ournal of

Probability and Statistics

Promensencen
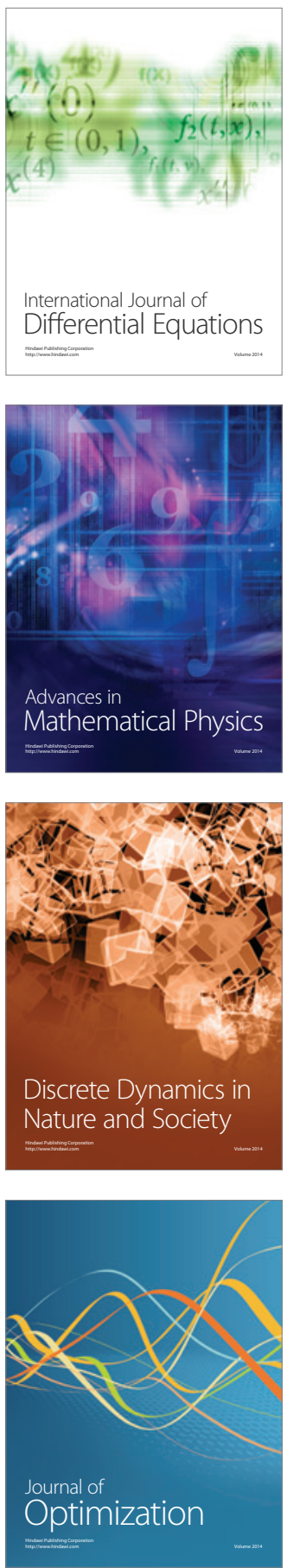